\title{
ANÁLISIS DEL AVANCE EN PRODUCTIVIDAD EN LA INDUSTRIA MANUFACTURERA MEXICANA, 1929-1944
}

\author{
JESÚS HUMBERTO ANGULO PALMERO \\ Benemérita Universidad Autónoma de Puebla ${ }^{a}$ \\ SYLVIA BEATRIZ GUILLERMO PEÓN \\ Benemérita Universidad Autónoma de Puebla
}

\begin{abstract}
RESUMEN
Este trabajo de investigación analiza el crecimiento en la productividad total de los factores (PTF) durante el período 1929-1944 y su importancia como fuente de crecimiento en la industria manufacturera mexicana. El análisis se realiza a nivel sectorial. La tarea principal del trabajo se enfoca en la estimación del crecimiento de la PTF, lo cual se lleva a cabo utilizando la metodología de Harberger denominada Método de Dos Deflactores, y cuya principal característica es que la cantidad de información requerida para calcular el crecimiento de la PTF es mucho más manejable -comparado con métodos tradicionales-, además de ser un método robusto y fácil de aplicar. Adicionalmente, el trabajo presenta un análisis empírico para apoyar la tesis de que el avance en la productividad fue una de las causas principales del proceso de sustitución de importaciones y del crecimiento observado en la industria manufacturera durante el período 1929-1939.
\end{abstract}

Palabras Clave: productividad total de los factores, crecimiento y productividad en México, productividad industrial manufacturera, método de dos deflactores.

${ }^{a}$ Facultad de Economía. Avenida San Claudio y 22 Sur. Colonia San Manuel. Puebla, Puebla, México.jangulo@ucla.edu; sguiller@eco.buap.mx 


\begin{abstract}
The paper analyzes total factor productivity (TFP) growth during the period 1929-1944 and its importance as a source of growth in Mexican manufacturing. The analysis of the industry is done at sector level. The paper task focuses on the estimation of TFP growth, and this is done using Harberger's methodology named the Two Deflator Method (2D), whose main characteristic is the realization that the amount of information required to compute the TFP growth rate is much more manageable compared to traditional methods, besides being a very robust and easily applicable method. In addition, the paper presents an empirical analysis to support the thesis that total factor productivity advance was one of the main causes to explain import substitution and growth in manufacturing during the period 1929-1939.
\end{abstract}

Keywords: total factor productivity, productivity and growth in Mexico, productivity in manufacturing, two deflator method

JEL Classification: O4, O47, N16

\title{
1. INTRODUCCIÓN
}

Uno de los componentes más importantes en la tasa de crecimiento del PIB para cualquier país es el crecimiento de la productividad o disminución de costos. Para el caso de México, la naturaleza de la información disponible sobre la actividad industrial ha ocasionado que los estudios sobre avances en la productividad hayan sido elaborados para períodos de tiempo posteriores a 1950, siendo los autores principales Enrique Cárdenas (1978) y Hernández Laos (1985). La metodología utilizada por Hernández y Cárdenas para sus estudios es muy similar a la diseñada por Griliches-Denison-Jorgenson (GDJ) ${ }^{1}$, metodología que ha demostrado ser demasiado exigente en cuanto a los requerimientos de información para lograr la estimación de los avances de la productividad. En la última década, Arnold C. Harberger ha encontrado en el denominado Método de Dos Deflactores (M2D) un método para estimar el avance en la productividad cuyos resultados han sido similares a los obtenidos por GDJ en cuanto a la calidad de la estimación se refiere; sin embargo, estos resultados se obtienen con la ventaja de que la cantidad de la información requerida es mucho menor y, por lo general, es información fácilmente disponible ${ }^{2}$.

\footnotetext{
${ }^{1}$ Denison (1967), Griliches (1963), Jorgenson (1967).

${ }^{2}$ Para una mejor explicación de las ventajas del método de los Dos Deflactores, véase Harberger (1998a).
} 
Es importante resaltar que, hasta la fecha, en la historia económica de México no ha sido analizado el tema de la productividad en la industria manufacturera para el período 1929-1944, y esto se debe principalmente a la falta de una metodología ad hoc para los datos disponibles para el período, por lo que podría decirse que, en lo que a productividad se refiere, existe una brecha en la historia económica mexicana para el período mencionado. Uno de los objetivos del presente trabajo de investigación es cerrar esta brecha en la historia económica de México, proporcionando un análisis sobre los avances en la productividad de la industria durante el período 1929-19443. Adicionalmente, se pretende aplicar y difundir la utilización del Método de Harberger de Dos Deflactores, cuya enorme ventaja consiste en que puede ser utilizado a cualquier nivel de agregación de la información, lo que facilita su aplicación en la estimación de la tasa de crecimiento de la productividad. El análisis de la tasa de crecimiento del PIB [del sector manufacturero] a través de la separación de sus componentes nos permitirá ahondar en el análisis de la anatomía del rápido crecimiento que México experimentó durante el período de estudio. Otro de los objetivos en esta investigación será presentar un análisis empírico que fundamente la hipótesis de que el crecimiento en el sector manufacturero durante 1929-1939 se debió al crecimiento de la productividad total de los factores, que, a su vez, incentivó el proceso de sustitución de importaciones durante la época. Esta hipótesis resulta diferente a la planteada por Cárdenas (1987), quien menciona que fue la política cambiaria de la época -cuyo objetivo era proporcionar protección al mercado interno- la que generó el proceso de sustitución de importaciones y, por tanto, el crecimiento. Así pues, en una primera instancia se realiza un análisis del crecimiento de la productividad total de los factores (PTF) en el sector manufacturero durante los años 1929-1944 para ponderar su importancia como fuente de crecimiento en el valor agregado industrial y en la expansión del mercado.

\section{ANTECEDENTES}

El proceso de industrialización en México comenzó alrededor de 1870, poco antes de que Porfirio Díaz iniciara su largo período como presidente del país (18761911), y fue precisamente durante los años que van de 1870 a 1910 cuando la industria mexicana experimentó un rápido proceso de crecimiento, particularmente

\footnotetext{
${ }^{3}$ Cabe aclarar que los censos industriales en México comenzaron a elaborarse en el año de 1929, por lo que el primer censo industrial fue publicado en 1930. Desde entonces, los censos industriales se realizan cada cinco años. En el período que nos ocupa, aunque los censos fueron publicados en 1930, 1935, 1940 y 1945, la información en realidad corresponde a los años de 1929, 1934, 1939 y 1944, respectivamente.
} 
en el sector de las manufacturas. Después de este período, la economía mexicana permaneció estancada hasta 1933, situación causada básicamente por la Revolución Mexicana (1910-1917) y la Gran Depresión ${ }^{4}$, la cual se experimentó en México durante el período 1926-1933. Sólo después de 1933 la economía mexicana, particularmente el sector manufacturero, reanudó su proceso de crecimiento rápido y sostenido, prolongándose éste hasta el año 1981.

El proceso de sustitución de importaciones comenzó a principios de los años treinta en México y se vio reforzado durante la Segunda Guerra Mundial (19391945). La mayoría de los estudiosos de la historia económica de México ${ }^{5}$ mencionan que más bien fue la política cambiaria del momento -la cual incentivó la sustitución de importaciones ${ }^{6}$-, y no el crecimiento en la PTF, la causa del rápido crecimiento de la industria manufacturera. A pesar de la importancia que el período 1929-1944 tiene para la historia económica de México, hay en la literatura muy pocos estudios sobre la industrialización mexicana para este período ${ }^{7}$, los más importantes de los cuales son los presentados por Cárdenas (1987) y Haber (1989). La investigación de Cárdenas sobre la industrialización en México se enfoca en el desarrollo de la industria en el contexto de la Gran Depresión, y describe las fuentes del crecimiento como provenientes tanto del lado de la oferta como de la demanda. De acuerdo con este autor, los principales factores de demanda que originaron el crecimiento industrial en México durante el período 1933-1939 fueron el

\footnotetext{
${ }^{4}$ Cárdenas (1987).

${ }^{5}$ Solís (1987).

${ }^{6}$ Cárdenas (1987), pp. 121-124.

${ }^{7}$ La razón por la que el período de análisis (1929-1944) ha sido casi inexplorado en la historia económica de México remite básicamente a que los datos disponibles para el análisis del proceso de industrialización son muy escasos y fragmentados. De todos los censos industriales elaborados durante el período, únicamente el de 1929 puede considerarse como completo, en el sentido de que es el único que incluye a todos los establecimientos y sectores industriales existentes durante ese período de tiempo en México. Los censos industriales subsecuentes -1934, 1939 y 1944- solamente incluyeron aquellos establecimientos que producían arriba de 10.000 pesos nominales, y por esta razón los datos y parámetros estimados para el crecimiento de la PTF no pueden ser directamente comparables en el tiempo, a menos que aquellos establecimientos que quedaron fuera de los censos subsecuentes al de 1929 realmente no tengan una participación representativa en la actividad productiva, o bien se encuentre una forma de corregir la influencia del problema de datos truncados -o perdidos. En relación con este problema, Angulo y Guillermo (2004) analizan los datos censales para cada sector -y a nivel subsector inclusive- para determinar la magnitud e importancia que tiene el ignorar el inconveniente de los datos truncados. El resultado del análisis de estos autores arroja que a nivel agregado -toda la industria manufacturera mexicana-, el número de establecimientos susceptibles de ser excluidos del censo de 1934 representó el 83 por cien de los reportados en 1929. Sin embargo, la producción proveniente de estos establecimientos excluidos no representó más del 4,29 por cien de la producción total de la industria manufacturera en 1934. Con esto puede concluirse que el peso o participación de los datos truncados o perdidos en el valor agregado de la industria manufacturera es demasiado pequeño como para sesgar significativamente las estimaciones de PTF y tasas de crecimiento sectoriales realizadas en el presente estudio.
} 
crecimiento en la demanda interna, la sustitución de importaciones y el aumento en las exportaciones. Por otra parte, entre los factores de oferta determinantes del crecimiento destaca el aprovechamiento de la capacidad instalada, la capacitación de la mano de obra y la PTF. En relación con esto último, Cárdenas presenta una estimación del crecimiento de la PTF del 1,12 por cien en promedio anual para la industria manufacturera durante el período 1929-1939, lo que explica un 26,1 por cien de la tasa promedio de crecimiento del producto manufacturero para ese mismo período. Por su parte, el estudio de Haber no elabora una nueva tesis acerca de las causas del crecimiento industrial para la época, sino que más bien trata de corroborar la hipótesis de Cárdenas de que los factores impulsores del crecimiento provienen principalmente de la demanda ${ }^{8}$. Sin embargo, a diferencia de Cárdenas, Haber utiliza datos provenientes de archivos de las empresas más grandes de la época y no utiliza información gubernamental (datos oficiales).

Las estimaciones sobre PTF en el presente artículo están basadas en datos directamente tomados de los censos y se realizan utilizando la metodología sugerida por Harberger denominada Método de Dos Deflactores o M2D ${ }^{9}$-el cual descompone la tasa de crecimiento del producto en crecimiento explicado por el incremento en el uso de capital, crecimiento explicado por el incremento en el uso de mano de obra y finalmente PTF-, lo que nos permitirá analizar la «anatomía» del rápido crecimiento industrial que México experimentó durante el período de análisis.

\section{PRODUCTIVIDAD TOTAL DE LOS FACTORES}

De acuerdo con la definición convencional, el crecimiento en la productividad total de los factores puede entenderse como aquella parte del crecimiento en el producto interno bruto (PIB) que no es explicada por el incremento en el uso y calidad de los factores de producción ${ }^{10}$, lo que también puede entenderse

\footnotetext{
${ }^{8}$ A este respecto es importante mencionar que la teoría económica moderna señala que el proceso de crecimiento económico involucra a la parte de la oferta de una economía, ya que es el lado de la oferta el que determina cuánto es físicamente capaz de producir esa economía. Así pues, podemos definir al crecimiento económico como el incremento de la capacidad productiva de un país a través del tiempo. Una mayor capacidad productiva es la clave para alcanzar mayores niveles de vida, ya que puede traducirse en mayor producto per cápita. Una mayor capacidad productiva permitirá a una economía lograr mayores niveles de producción sin detonar la inflación.

${ }^{9}$ Harberger (1998a).

${ }^{10}$ Aunque es un concepto económico básico, cabe recordar aquí que el concepto de productividad total de los factores es diferente del concepto de productividad marginal de un factor de producción. Así, por ejemplo, la productividad marginal del capital se refiere al incremento en el producto como respuesta al incremento en el uso del factor capital $-\mathrm{y}$ la definición análoga se aplica al factor trabajo.
} 
como el crecimiento en el producto que ocurre cuando el cambio en el uso cuantitativo y cualitativo de los factores de producción es cero. Abramovitz (1962, pp. 762-766) define a la PTF como el efecto gratuito del avance en tecnología aplicada, eficiencia administrativa y organización industrial. El concepto de gratuito es interpretado por este autor como todo aquel efecto que se obtiene por encima del costo de esos factores, el cual puede entenderse, a su vez, como el empleo de los recursos escasos con usos alternativos.

La mayoría de los economistas percibe el crecimiento de la PTF como un desplazamiento de la función producción, y estos desplazamientos se asocian con nuevas tecnologías, inventos, o como el resultado de gastos en investigación y desarrollo. Sin embargo, el crecimiento de la PTF puede manifestarse como un desplazamiento de la función producción y como un movimiento a lo largo de la misma función producción. De acuerdo con Jorgenson (1967, pp. 313-314), el crecimiento de la PTF puede identificarse como un desplazamiento en la función producción si ésta presenta rendimientos constantes a escala y si existe equilibrio en el mercado de insumos - es decir, si la tasa marginal de sustitución es igual a la razón de precios. Pero si la función producción incorpora los efectos de rendimientos crecientes a escala, externalidades y desequilibrio en el mercado de insumos, entonces los cambios en la PTF pueden interpretarse como movimientos a lo largo de la función producción. Se trate de movimientos a lo largo de la función producción o de desplazamientos de la misma, lo cierto es que, para la gente involucrada en la actividad productiva -los hombres de negocios-, es difícil percibir y describir una función de producción de su empresa o compañía. Para un empresario es mucho más fácil percibir el crecimiento de la PTF como todas aquellas actividades que tienen por efecto el reducir los costos en la producción de bienes y servicios. Esta es la forma en que Harberger (1998a,1998b) percibe el crecimiento de la PTF: «reducción en costos reales». En este sentido, resulta lógico ubicar el origen del crecimiento de la PTF a nivel de la empresa -más que a nivel agregado. Para Harberger, son los hombres de negocios, los administradores y los jefes de producción los agentes clave en la tarea descubrir e implementar actividades encaminadas a lograr reducción en costos reales [incrementos en PTF]. Pero, si bien es cierto que el crecimiento de la PTF tiene su origen en la empresa, no podemos descartar el análisis de este importante concepto a niveles de mayor agregación económica. Los economistas debemos procurar la búsqueda e implementación de aquellas políticas que promuevan el crecimiento económico, y si el crecimiento de la PTF es una parte muy importante del crecimiento económico, resulta de especial interés ahondar en el análisis de la evolución de la PTF a nivel sectorial. 


\subsection{Estimación de la PTF}

Algebraicamente, la PTF se representa como sigue:

$$
P T F_{j t}=\frac{\Delta y_{j t}}{y_{j t-1}}-\frac{\left(\rho_{j t}+\delta_{j t}\right) \Delta K_{j t}}{y_{j t-1}}-\frac{w_{t}^{*} \Delta L_{j t}^{*}}{y_{j t-1}}
$$

donde:

$P T F_{j t}=$ Productividad total de los factores en el sector industrial $j$, periodo $t$

$\Delta y_{j t} / y_{j t-1}=$ tasa de crecimiento ${ }^{11}$ en el producto real (valor agregado) para el sector industrial $j$, periodo $t$

$\rho_{j t}=$ tasa neta de rendimiento del capital del sector industrial $j$, periodo $t$

$\delta_{i t}=$ tasa neta de depreciación del capital del sector industrial $j$, periodo $t$

$\left(\rho_{j t}+\delta_{i t}\right) D K_{j t} / y_{j t-1}=$ contribución del capital a la tasa de crecimiento en el sector industrial $j$, periodo $t$

$w^{*}{ }_{t} \Delta L^{*}{ }_{j t} / y_{j t-1}=$ contribución de la mano de obra a la tasa de crecimiento del sector industrial $j$, periodo $t$.

La estimación de la PTF en el presente trabajo de investigación se realizó a nivel de sector -para doce sectores de la industria manufacturera- utilizando el M2D ${ }^{12}$. El primer paso para la aplicación del método es la estimación de $\rho$, es decir, la tasa de rendimiento sobre el capital (TRC), definida ésta como el ingreso imputable al capital, en proporción al acervo de capital. El crecimiento en el producto imputable al incremento en el factor capital -o contribución del capital a la tasa de crecimiento-, puede entonces representarse como $(\rho+\delta) \Delta K / y$. La segunda parte del M2D se enfoca en la estimación del ingreso imputable al crecimiento de la mano de obra -crecimiento en número de unidades de trabajo utilizadas-, así como también en la estimación del ingreso imputable a la acumulación de capital humano -por ejemplo experiencia, educación, capacitación, etc. La separación de estas dos últimas fuentes de ingreso nos permite evitar el contabilizar la contribución que el mejoramiento en la mano de obra tiene en la productividad del trabajo como

\footnotetext{
${ }^{11}$ Se refiere a la variación porcentual registrada por la variable entre el período $t-1 \mathrm{y} t$.

12 Para demostrar la validez del M2D, Harberger, realiza estimaciones de tasas de crecimiento de la PTF para 33 sectores de la industria manufacturera norteamericana, con datos anuales de 1948 a 1991, utilizando su metodología y también la metodología tradicional de Jorgenson y sus colaboradores. Los resultados muestran claramente la gran similitud de ambos métodos de estimación. En palabras de Harberger (1998a, pp. 47), «[...] el M2D es un método burdo. Pero también es un método tremendamente robusto y fácilmente aplicable». Por su parte, Edgar Robles (1997) utiliza también el M2D y realiza comparaciones de sus estimaciones con las obtenidas al aplicar el método tradicional para el cálculo de tasas de crecimiento de la PTF en veinte sectores de la industria manufacturera norteamericana durante el período 1948-1979. Sus resultados arrojan también mucha similitud entre los dos métodos.
} 
parte del incremento de la PTF. Para poder estimar la contribución de la mano de obra en el crecimiento a través del M2D necesitamos seleccionar un salario real representativo $\left(w^{*}\right)$ para la mano de obra relativamente «no cualificada» (trabajador tipo). Usando $w^{*}$, podemos entonces atribuir al cambio en cualquier categoría dada de mano de obra $\Delta L_{i j}$, una contribución al crecimiento en el producto $\left(y_{i}\right)$ igual a $w^{*}$ —por la mano de obra sin cualificación — más $\left(w_{i j}^{*}-w^{*}\right)$-por su contribución de capital humano al crecimiento en el producto-, multiplicado esto por el cambio en el número de trabajadores $\Delta L_{i j}$.

\section{Estimación de la TRC: el papel del primer deflactor}

La estimación de la TRC es necesaria para poder calcular la contribución del capital a la tasa de crecimiento del producto. Para medir la TRC, ambos, el numerador - pesos reales de rendimiento- y el denominador -acervo de capital-, deben estar expresados en las mismas unidades. Siguiendo el M2D de Harberger, la forma más efectiva de hacer esto es midiendo el producto -valor agregado- y el acervo de capital en unidades del deflactor del PIB. Este es precisamente el papel del primer deflactor en el M2D. Por definición, la tasa de rendimiento de capital (TRC) bruta $(r+\delta)$ puede estimarse restándole al producto real los pagos totales —en términos reales - realizados a otros insumos diferentes del capital, y dividiendo este resultado entre el acervo de capital en términos reales. Esto es:

donde $y_{j t}$ es el producto real en el sector $j$ en el periodo $t, w_{j t} L_{j t}$ es el pago real a la mano de obra en el sector $j$, periodo $t, r m_{j t}$ es el pago real en materia prima utilizada en el proceso de producción del sector $j$ en el periodo $t$, $\delta_{j}$ es la tasa real de depreciación ${ }^{13}$ del capital en el sector $j$, y $K_{j t}$ es el acervo de capital de el sector $j$ en el periodo $t$. En el presente trabajo, algunos de los elementos de la ecuación [2] pueden ser directamente obtenidos de los censos, pero algunos otros necesitan ser calculados. En particular, en el cálculo de la TRC son considerados los siguientes insumos: mano de obra, capital, materia prima, y otros -por ejemplo, electricidad, lubricantes, combustibles, etc. Para el caso del acervo de capital, los censos informan sobre esta variable desagregada de la siguiente forma: valor de la maquinaria, inventarios de materia prima y producto final, y valor de edificios y terrenos utilizados en el proceso de producción. Sin embargo, cabe notar aquí que los activos reportados pueden estar infravalorados. Podrían mencionarse muchas razones por las que este problema de subvaloración del capital puede estar presente

\footnotetext{
${ }^{13}$ En este caso, $\delta_{j}$ es la tasa de depreciación promedio para todos los activos del sector $j$.
} 
en el caso de los censos mexicanos, pero probablemente la más sensata es que el acervo de capital reportado por las empresas en cada industria es el valor en libros de los activos, el cual obviamente está basado en políticas contables y no necesariamente refleja el valor económico o valor de mercado de los activos. El valor económico del capital está relacionado con la capacidad del mismo para generar flujos de efectivo. En México, la maquinaria e instalaciones de empresas por lo general tienen una vida productiva más larga, es decir, se usan y explotan por un periodo de tiempo más prolongado en comparación con lo que es un tiempo de utilización común en países industriales como Estados Unidos ${ }^{14}$. Esto nos sugiere que muchos activos con valor en libros de cero aún están en uso y aún están produciendo y generando ingresos, por lo cual aún tienen un valor económico positivo. Haber (1989, pp. 177) en sus investigaciones encontró una baja tasa de utilización de la capacidad instalada en la industria mexicana, lo que generalmente se asocia con una baja tasa de depreciación de los activos. En particular, este autor presenta indicadores de utilización de la capacidad instalada en las industrias del cemento y el acero (1933-1938), y en promedio el índice de utilización -producción dividida por capacidad instalada- es bajo a pesar del boom que estas industrias estuvieron experimentando como consecuencia del incremento en el gasto del Gobierno en infraestructura para ese tiempo. Bajas tasas de utilización de la capacidad instalada deben llevar a bajas tasas de depreciación de esos activos. Esta es sólo una de las razones por las que la valoración del acervo de capital debe ser corregida en el caso de México. Para propósitos del presente trabajo de investigación, la metodología utilizada en la corrección del valor del acervo de capital se basa en la encuesta de acervos y formación de capital de Banco de México ${ }^{15}$. Otro ele-

\footnotetext{
${ }^{14}$ En países industriales como Estados Unidos, el valor en libros de los activos por lo general es más cercano a su valor económico. La depreciación que se muestra en la contabilidad de las empresas en este caso es más representativa de la vida útil de un activo, indicando cuándo la maquinaria o instalaciones realmente necesitan ser reemplazadas por nuevas tecnologías -usualmente superiores en productividad.

${ }^{15}$ El método más usado para la determinación del acervo de capital -sobre todo a nivel agregado- es el conocido como Método de Inventarios Perpetuos. El acervo de capital en $t$, se halla acumulando la inversión neta año tras año $(t-1, t-2, \ldots, t-k)$. Sin embargo, debe considerarse que para el cálculo del acervo de capital en $t$, la inversión del pasado cobra menos relevancia mientras más lejana se encuentre del presente $(t)$ - es decir, mientras mayor sea $k$. En la práctica, el método de inventarios perpetuos deflacta la inversión nominal por un determinado deflactor previamente elegido, y al resultado se le agrega el acervo de capital real del inicio del período después de haber ajustado por la tasa de depreciación $\delta$-económica y no contable. Es decir, para cada $t$ el acervo de capital sería $k_{t}=k_{t-1}(1-\delta)+I_{t}$, donde $k$ e $I$ están expresados en las mismas unidades, es decir en términos del mismo deflactor. Ahora bien, al utilizar los datos censales no se tiene forma de conocer la inversión año con año. Sin embargo, en este mismo sentido y siguiendo el espíritu del método de inventarios perpetuos para calcular el acervo de capital real bruto, se utiliza la siguiente ecuación:
}

$$
k^{*}=\left(\frac{1}{55}\right) \frac{k_{t}}{P_{t-10}}+\left(\frac{2}{55}\right) \frac{k_{t}}{P_{t-9}}+\left(\frac{3}{55}\right) \frac{k_{t}}{P_{t-8}}+\ldots+\left(\frac{10}{55}\right) \frac{k_{t}}{P_{t}}
$$


mento a ser calculado para la estimación de la TRC a través de la ecuación [2] es el gasto total en materias primas $\left(r m_{j t}\right)$, cuya forma de cálculo se especifica en el apéndice. Con relación al insumo trabajo, los censos contienen información bastante completa y sensata sobre salarios, y se presenta información desagregada para tres categorías diferentes de la mano de obra: directores -los que toman las decisiones en la empresa-, empleados y obreros. Finalmente, todas las variables incluidas en la ecuación [2] son expresadas en términos reales utilizando el deflactor implícito del PIB. Este último es un paso indispensable en el cálculo de la TRC, ya que se requiere expresar el numerador y el denominador de la TRC en las mismas unidades. De acuerdo con Harberger (1998a, pp. 10), las unidades en las que se expresa al acervo de capital en el M2D representan una diferencia clave de este método con relación al enfoque tradicional -utilizado por Jorgenson, Grilliches y Denison-, el cual visualiza al acervo de capital real como cantidades de máquinas, edificios e inventarios, mientras que el M2D visualiza al capital real como una cantidad de poder adquisitivo real que ha sido asignada con el propósito de generar ingreso futuro ${ }^{16}$. Como el mismo Harberger (1998a, pp. 10-11) menciona,

«Es importante darse cuenta de que este atributo del M2D no es una aberración. Esta es exactamente la forma en que el mismo problema se ha venido manejando siempre en el análisis de inversiones, donde los flujos de efectivo de un proyecto de inversión son puestos en términos reales a través de deflactar todos los flujos (los negativos correspondientes a los años de inversión, y los flujos positivos correspondientes a los años de producción) por el mismo deflactor. Para el caso de la tasa real de rendimiento del capital, el flujo de ingreso del capital (numerador) debe estar expresado en las mismas unidades reales que el denominador (el acervo de capital). De la misma forma, para calcular el valor presente neto real de un proyecto, todos los flujos de beneficios y costos deben denominarse en las mismas unidades reales» ${ }^{17}$.

$\overline{\text { donde } k_{t}}$ * es acervo de capital real bruto estimado, $k_{t}$, son los activos totales brutos reportados en el censo y $P_{t}$ es el deflactor implícito del PIB. La ecuación anterior supone que el acervo de capital actual se formó a través del tiempo. En este sentido una fracción (1/55) de los activos totales de hoy fueron adquiridos hace 10 años, y una fracción (2/55) de los activos totales de hoy, se adquirieron hace 9 años, etc. Por supuesto, los activos adquiridos más atrás en el tiempo tienen menos peso en el valor del capital actual.

${ }^{16} \mathrm{El}$ enfoque alternativo para deflactar el acervo de capital sería obtener datos de precios de todos los activos de capital específicos utilizados por cada sector -o quizás subsector- que está bajo estudio. Obviamente, este procedimiento resulta muy complicado y abierto a serios errores cuando se trata de estimar tal deflactor, particularmente cuando los datos se encuentran agregados por sectores o subsectores.

${ }^{17}$ Traducción de los autores. 
Estimación de la contribución del factor trabajo al crecimiento: el papel del segundo deflactor

La contribución de la mano de obra al crecimiento del producto de la industria $i$ en el periodo $t$, está dada por el término $\left(w^{*} t L_{j t}^{*} / y_{j t}\right)$ en la ecuación [1]. Para captar la gran heterogeneidad del factor trabajo es necesario contar con una descomposición detallada de las diferentes categorías de la mano de obra. La contribución de la mano de obra es entonces $\sum_{i} w_{i j, t} \Delta L_{i j, t}$ donde $w_{i j, t}$ representa el salario real para un trabajador de la categoría $i$, en el sector $j$ y el periodo $t$, y $\Delta L_{i j, t}$ representa el cambio en horas trabajadas en la categoría $i$, en el sector $j$ en el periodo $t$. Sin embargo, el número relevante de categorías para la mano de obra puede ser bastante grande, lo que convierte esta tarea en más complicada a medida que las categorías de mano de obra se encuentren más desagregadas. Para evitar tal complicación en el cálculo de la contribución de la mano de obra al crecimiento, el M2D utiliza un salario estándar $w^{*}$ asignado a la mano de obra estándar o mano de obra «no cualificada» ${ }^{18}$, y, tal y como explica Harberger (1998b, pp. 29), el salario en exceso sobre $w^{*}$ para cualquier trabajador es lo atribuible al capital humano. Los rendimientos provenientes de la educación, entrenamiento, capacitación y experiencia quedan incluidos en este «salario en exceso» bajo esta interpretación. La variable $w^{*}$ es de hecho el segundo deflactor en el M2D y tiene el propósito de evitar la enorme complicación que involucra el encontrar la contribución de la mano de obra al crecimiento cuando se utiliza el método de JorgensonGriliches, ya que no hay necesidad de dividir la fuerza laboral en un número grande de categorías -hombre, mujer, joven, viejo, experiencia, etc. El salario del trabajador relativamente no cualificado $\left(w^{*}\right)$, se utiliza entonces como un numerario en el sentido de que, si tenemos el número de horas trabajadas $(h)$ para cierto individuo, y si su salario por hora es $w$, entonces $h w^{*}$ serán sus ingresos puros provenientes del trabajo y $\left(w-w^{*}\right) h$ son sus ingresos imputables al capital humano que posee. En el presente trabajo de investigación, el cálculo del segundo deflactor $\left(w^{*}\right)$ se desarrolla dividiendo la nómina total pagada a los obreros en el sector industrial $j$, en el periodo $t$, entre el número de obreros en el mismo sector industrial y periodo de tiempo, lo que nos da $w_{j i t}^{*}$. Ambas variables -número de obreros y nómina pagada- son datos que se obtienen directamente de los censos. Una vez que se obtiene $w^{*}{ }_{j t}$ para cada sector industrial, $w^{*}$ (segundo deflactor) se calcula como la mediana de todos los sectores industriales para el momento $t$. Algebraicamente,

$$
w_{j t}^{*}=\frac{\text { nomobr }_{j t}}{o b r_{j t}} \mathrm{y} w_{t}^{*}=\operatorname{Mediana}\left(w_{j t}^{*}\right)
$$

${ }^{18}$ Nos referimos aquí a aquellos trabajadores que no cuentan con preparación para la realización de un determinado trabajo. 
donde nomobr $r_{j t}$ es la nómina pagada a los obreros en el sector $j$, periodo $t$, y $o b r_{j t}$ es el número de obreros en el sector $j$ en el periodo $t$. El siguiente paso en la estimación de la PTF corresponde al cálculo de $L_{j i}^{*}$, el cual se realiza directamente, ya que únicamente se requiere dividir la nómina total de cada sector en el periodo $t$ entre $w_{t}^{*}$-el deflactor de la mano de obra. Esto es $L_{j t}^{*}=$ Nómina Total ${ }_{\mathrm{jt}} / w_{t}{ }_{t}$. Hasta aquí se han explicado con algo de detalle los procedimientos utilizados para la estimación de la PTF para cada sector industrial en nuestro estudio, por lo que el siguiente paso será la descripción y análisis de la PTF agregada, para lo que se utilizará la siguiente ecuación:

$$
\Delta P T F_{t}^{*}=\sum_{j=1}^{N}\left[\left(\frac{y_{j t-1}}{y_{t-1}}\right) \Delta P T F_{j t}\right] \text { [3] }
$$

donde $\Delta P T F_{t}^{*}$ es el cambio porcentual en la PTF agregada, en el periodo $t, \mathrm{y} \Delta P T F_{j t}$ es el cambio porcentual en la PTF del sector $j$, periodo $t$, el cual es ponderado por su participación en el valor agregado total. La ecuación [3] servirá de base para la construcción del diagrama de productividad denominado Sunset-Sunrise, el cual se explicará mas adelante.

\section{RESULTADOS DE LAS ESTIMACIONES POR SECTORES}

Para el presente trabajo se utilizaron datos procedentes de los censos de 1929, 1934, 1939 y 1944. Los censos industriales contienen los datos de producto, número de trabajadores, salarios, acervo de capital, etc., de los principales sectores de la industria manufacturera mexicana, entre los cuales se encuentran los siguientes: alimentos y bebidas, tabaco, textiles, imprenta y publicación, cuero y pieles, química, ropa, madera y muebles, papel, construcción y materiales, cerámica y vidrio, fundición y manufactura de artículos metálicos. Con los datos obtenidos de los censos puede calcularse la evolución de la PTF para la industria manufacturera mexicana -abarcando 12 sectores- utilizando el M2D, aunque debe tomarse en cuenta que solamente el censo de 1929 reporta datos completos (no truncados). Sin embargo, la magnitud del sesgo depende de la importancia que la parte truncada tenga en la producción total de cada sector y en la industria manufacturera. A este respecto cabe recordar que, como indicábamos más atrás, el peso de la información truncada en el censo de 1934 es demasiado pequeño como para poder sesgar las estimaciones de manera significativa. La estimación de la PTF se llevó a cabo, pues, a nivel de sector, utilizando los datos de los censos industriales. Los doce sectores de la industria manufacturera incluidos representan alrededor del 90 por cien del valor bruto de la producción total para los diferentes años censales. El valor agregado real para el conjunto de sectores analizados decreció 
durante el período que va de 1929 a 1934 en un 11,93 por cien -es decir, una caída de 2,39 por cien en promedio anual. Esto sucedió a pesar de que la depresión habría tocado fondo desde 1933, y para 1934 la economía mexicana experimentaba ya una franca recuperación alcanzándose los niveles de 1929 en la producción real manufacturera ${ }^{19}$. Por supuesto, la situación de la industria también se refleja en las estimaciones de PTF a nivel de sector y a nivel de industria (agregado). El avance en productividad -crecimiento promedio anual de la PTF- en conjunto para los sectores analizados en el período 1929-1934 fue de 3,23 por cien, es decir, la economía mexicana experimentó en este período una reducción en costos o avance en productividad. Los promedios anuales de avance en productividad por sector para ese periodo se muestran en el Cuadro 1, columna F. Es necesario mencionar que, para llegar a estos resultados, fue necesario realizar algunas correcciones en los datos, ya que varios subsectores no reportaron el rubro de materias primas utilizadas en el proceso productivo en el censo realizado en 1929, e ignorar este problema resultaría en la sobreestimación del valor agregado de los sectores correspondientes. Las correcciones se detallan en el apéndice presentado al final del artículo. Los cuadros 2 y 3 muestran las correspondientes tasas de crecimiento anual promedio de la PTF para los periodos 1934-1939 y 1939-1944, respectivamente. Los resultados que se presentan son consistentes con la hipótesis de que el proceso de industrialización en México se reinicia en el período post gran depresión.

\subsection{Diagramas de Productividad Sunset-Sunrise para la industria manufacture- ra en México ${ }^{20}$}

Harberger (1998b) ha propuesto una forma innovadora de visualizar gráficamente la distribución de productividad entre sectores industriales. Primeramente se ordenan los sectores de acuerdo con su productividad, tomando en primer lugar aquel sector con mayor tasa de crecimiento de la PTF. La contribución de la PTF de cada sector al crecimiento total de la industria se calcula multiplicando su correspondiente tasa de crecimiento de la PTF por su participación en el valor agregado (VA). La columna $\mathrm{F}$ en el Cuadro 1 presenta las contribuciones acumuladas de la PTF al crecimiento para cada industria adicional. El diagrama de productividad Sunrise se crea al representar gráficamente las dos últimas columnas del

${ }^{19}$ Solís (1987).

${ }^{20} \mathrm{El} \mathrm{nombre} \mathrm{del} \mathrm{diagrama} \mathrm{se} \mathrm{atribuye} \mathrm{a} \mathrm{la} \mathrm{forma} \mathrm{que} \mathrm{toma} \mathrm{la} \mathrm{productividad} \mathrm{agregada,} \mathrm{y} \mathrm{su}$ similitud con una «puesta de sol» o «salida de sol», dependiendo de la contribución de las industrias a la PTF agregada. Si la tasa de crecimiento en la PTF agregada es positiva, el resultado será un diagrama sunrise, mientras que, si la tasa de crecimiento agregada de la PTF es negativa, se obtendrá un diagrama sunset. 
Cuadro 1. Puede verse en el diagrama de productividad una similitud cercana a la curva de Lorenz, por lo que a continuación se explica. El crecimiento de la PTF agregada para todos los sectores de la industria manufacturera en el período 19291934 resultó ser 3,23 por cien -en promedio anual-, y la magnitud y distribución de tasas de crecimiento de la PTF por sector es lo que determina la forma del diagrama de productividad, que en este caso toma la forma de sunrise o salida de sol (véase Gráfico 1). La parte de la curva que presenta pendiente creciente es resultado de las contribuciones acumuladas de aquellos sectores con crecimiento de la PTF positivo, mientras que la parte con pendiente decreciente es resultado de las contribuciones acumuladas de los sectores con productividad negativa. Puede notarse en la columna F del Cuadro 1 que la tasa máxima de crecimiento de la PTF fue 3,91 por cien. Esta es la tasa de crecimiento que podría haberse logrado si los sectores con productividad negativa fueran eliminados de la economía. Resulta interesante notar también que los sectores con tasas de crecimiento positivo de la PTF en conjunto produjeron el 82,93 por cien del VA total de la industria manufacturera, y que el restante 17 por cien del VA fue producido por sectores que presentaron tasas de crecimiento negativas en PTF, lo que implica una caída en productividad -o aumento en costos reales. La industria manufacturera en su conjunto pudo haber alcanzado una tasa de crecimiento de casi el 4 por cien de la PTF promedio anual durante el quinquenio 1929-1934, si hubiera sido posible eliminar las contribuciones negativas de los sectores improductivos -que en este período resultaron ser solamente dos: imprenta y publicidad y fundición. Es de notar también que dos de los sectores productivos incluidos -textiles y alimentos y bebidas- son grandes sectores para la época, con una contribución importante en la productividad de la industria como un todo. Otro punto de interés a señalar en el diagrama de productividad es el punto $A^{21}$, el cual indica que solamente con el primer 46 por cien de participación en el VA se alcanza el crecimiento de la PTF agregado en la industria manufacturera, que fue del 3,23 por cien. Con este análisis podemos darnos cuenta de que el diagrama de productividad resulta ser una representación visual clara del grado de concentración o dispersión del avance en productividad -o reducción en costos reales- entre los componentes de un agregado: en este caso, los sectores que componen la industria manufacturera.

Por otra parte, resulta importante mencionar que, durante el período 1929-1934, el valor agregado de la industria manufacturera -los doce sectores incluidos-, tuvo una tasa de crecimiento promedio anual de 4,2 por cien. El crecimiento promedio anual de la PTF agregada para la industria en este período fue de 3,23 por cien (véase Cuadro 1), lo que significa que el 76,9 por cien del crecimiento en el producto de manufacturas es explicado por avances en la productividad. Este es un

${ }^{21}$ El punto A queda ubicado al trazar una línea horizontal sobre el gráfico, al nivel de la tasa de PTF agregada -en este caso, 3,23 por cien. La intersección de esta línea horizontal con la curva de productividad, nos indica con qué porcentaje de VA en la economía se habría alcanzado la tasa de crecimiento agregada de la PTF. 
resultado bastante razonable si consideramos que en ese entonces la economía salía de un período de depresión en el que se tenía una gran capacidad ociosa en la planta productiva ${ }^{22}$ heredada de los años veinte. La utilización de esta capacidad ociosa a principios de la década de los treinta puede entenderse como parte de este avance en la productividad. Para el período 1934-1939, el VA de la industria manufacturera creció aproximadamente el 7,9 por cien en promedio anual. La tasa de crecimiento promedio anual de la PTF para este lustro (véase Cuadro 2) fue de 3,72 por cien. Esto significa que 47,08 por cien de la tasa de crecimiento anual del VA es explicada por los avances en productividad -o reducción en costos reales- en la industria manufacturera. A la luz de la información proporcionada por estos dos períodos quinquenales (1929-1939), podemos analizar el crecimiento de la PTF con el objetivo de demostrar que éste fue una de las fuentes principales del crecimiento económico observado durante el período.

\section{CUADRO 1}

CRECIMIENTO PROMEDIO ANUAL DE LA PTF POR SECTOR INDUSTRIAL Y SU CONTRIBUCIÓN AL CRECIMIENTO DE LA PTF AGREGADA, 1929-1934

\begin{tabular}{|l|c|c|c|c|c|}
\hline \multicolumn{1}{|c|}{$\begin{array}{c}\text { A } \\
\text { Industrial }\end{array}$} & $\begin{array}{c}\text { B } \\
\text { Tasa de } \\
\text { Crecimiento } \\
\text { de la PTF }\end{array}$ & $\begin{array}{c}\text { C } \\
\text { Participación } \\
\text { en el Valor } \\
\text { Agregado }\end{array}$ & $\begin{array}{c}\text { D } \\
\text { Contribución } \\
\text { de la PTF al } \\
\text { Crecimiento } \\
\text { (B*C) }\end{array}$ & $\begin{array}{c}\text { E } \\
\text { Participación } \\
\text { acumulada } \\
\text { en el V.A. }\end{array}$ & $\begin{array}{c}\text { E } \\
\text { Contribución } \\
\text { acumulada } \\
\text { de la PTF al } \\
\text { crecimiento }\end{array}$ \\
\hline $\begin{array}{l}\text { Cerámica y } \\
\text { Vidrio }\end{array}$ & $13,14 \%$ & $0,80 \%$ & $0,11 \%$ & $0,80 \%$ & $0,11 \%$ \\
\hline Tabaco & $8,98 \%$ & $5,34 \%$ & $0,48 \%$ & $6,14 \%$ & $0,58 \%$ \\
\hline $\begin{array}{l}\text { Madera y } \\
\text { Muebles }\end{array}$ & $7,85 \%$ & $4,46 \%$ & $0,35 \%$ & $10,60 \%$ & $0,93 \%$ \\
\hline Química & $8,11 \%$ & $4,02 \%$ & $0,33 \%$ & $14,62 \%$ & $1,26 \%$ \\
\hline $\begin{array}{l}\text { Cuero y } \\
\text { Pieles }\end{array}$ & $6,68 \%$ & $1,53 \%$ & $0,10 \%$ & $16,15 \%$ & $1,36 \%$ \\
\hline Textiles & $6,50 \%$ & $25,38 \%$ & $1,65 \%$ & $41,53 \%$ & $3,01 \%$ \\
\hline $\begin{array}{l}\text { Construcción } \\
\text { y Materiales }\end{array}$ & $4,25 \%$ & $2,43 \%$ & $0,10 \%$ & $43,96 \%$ & $3,12 \%$ \\
\hline Papel & $3,45 \%$ & $1,96 \%$ & $0,07 \%$ & $45,92 \%$ & $3,18 \%$ \\
\hline $\begin{array}{l}\text { Alimentos y } \\
\text { Bebidas }\end{array}$ & $2,30 \%$ & $30,13 \%$ & $0,69 \%$ & $76,05 \%$ & $3,88 \%$ \\
\hline $\begin{array}{l}\text { Ropa y } \\
\text { Calzado }\end{array}$ & $0,49 \%$ & $6,88 \%$ & $0,03 \%$ & $82,93 \%$ & $3,91 \%$ \\
\hline $\begin{array}{l}\text { Imprenta y } \\
\text { Publicidad }\end{array}$ & $-3,40 \%$ & $4,66 \%$ & $-0,16 \%$ & $87,59 \%$ & $3,75 \%$ \\
\hline Fundición & $-4,17 \%$ & $12,41 \%$ & $-0,52 \%$ & $100,00 \%$ & $3,23 \%$ \\
\hline
\end{tabular}

Fuente: cálculos propios utilizando datos censales.

\footnotetext{
${ }^{22}$ Cárdenas (1978).
} 


\section{CUADRO 2}

CRECIMIENTO PROMEDIO ANUAL DE LAPTF POR SECTOR INDUSTRIAL Y SU CONTRIBUCIÓN AL CRECIMIENTO EN LAPTF AGREGADA, 1934-1939

\begin{tabular}{|l|c|c|c|c|c|}
\hline \multicolumn{1}{|c|}{$\begin{array}{c}\text { A } \\
\text { Sector } \\
\text { Industrial }\end{array}$} & $\begin{array}{c}\text { B } \\
\text { Tasa de } \\
\text { Crecimiento } \\
\text { de la PTF }\end{array}$ & $\begin{array}{c}\text { C } \\
\text { Participación } \\
\text { en el Valor } \\
\text { Agregado }\end{array}$ & $\begin{array}{c}\text { D } \\
\text { Contribución } \\
\text { de la PTF al } \\
\text { Crecimiento } \\
\text { (B*C) }\end{array}$ & $\begin{array}{c}\text { E } \\
\text { Participación } \\
\text { acumulada } \\
\text { en el V.A. }\end{array}$ & $\begin{array}{c}\text { E } \\
\text { Contribución } \\
\text { acumulada } \\
\text { de la PTF al } \\
\text { crecimiento }\end{array}$ \\
\hline $\begin{array}{l}\text { Alimentos y } \\
\text { Bebidas }\end{array}$ & $6,86 \%$ & $26,21 \%$ & $1,80 \%$ & $26,21 \%$ & $1,80 \%$ \\
\hline Fundición & $6,45 \%$ & $3,96 \%$ & $0,26 \%$ & $30,16 \%$ & $2,05 \%$ \\
\hline Textiles & $4,55 \%$ & $33,85 \%$ & $1,54 \%$ & $64,02 \%$ & $3,59 \%$ \\
\hline Papel & $4,41 \%$ & $2,24 \%$ & $0,10 \%$ & $66,26 \%$ & $3,69 \%$ \\
\hline $\begin{array}{l}\text { Imprenta y } \\
\text { Publicidad }\end{array}$ & $4,16 \%$ & $3,30 \%$ & $0,14 \%$ & $69,55 \%$ & $3,83 \%$ \\
\hline $\begin{array}{l}\text { Ropa y } \\
\text { Calzado }\end{array}$ & $3,23 \%$ & $6,87 \%$ & $0,22 \%$ & $76,42 \%$ & $4,05 \%$ \\
\hline $\begin{array}{l}\text { Madera y } \\
\text { Muebles }\end{array}$ & $2,51 \%$ & $3,57 \%$ & $0,09 \%$ & $79,99 \%$ & $4,14 \%$ \\
\hline $\begin{array}{l}\text { Construcción } \\
\text { y Materiales }\end{array}$ & $0,68 \%$ & $3,70 \%$ & $0,03 \%$ & $83,69 \%$ & $4,17 \%$ \\
\hline Química & $0,49 \%$ & $6,60 \%$ & $0,03 \%$ & $90,29 \%$ & $4,20 \%$ \\
\hline $\begin{array}{l}\text { Cuero y } \\
\text { Pieles }\end{array}$ & $-1,30 \%$ & $1,35 \%$ & $-0,02 \%$ & $91,65 \%$ & $4,18 \%$ \\
\hline $\begin{array}{l}\text { Cerámica y } \\
\text { Vidrio }\end{array}$ & $-2,15 \%$ & $1,71 \%$ & $-0,04 \%$ & $93,36 \%$ & $4,14 \%$ \\
\hline Tabaco & $-6,40 \%$ & $6,64 \%$ & $-0,43 \%$ & $100,00 \%$ & $3,72 \%$ \\
\hline
\end{tabular}

Fuente: cálculos propios utilizando datos censales.

En el siguiente apartado se presentará evidencia para demostrar que los avances en productividad experimentados en la industria manufacturera fueron una de las causas principales que indujeron la sustitución de importaciones durante el período, y no lo fue la devaluación del tipo de cambio nominal -que resultó también en una devaluación del tipo de cambio real en este período-, como se ha dicho hasta ahora en la literatura.

La reducción en costos reales [avance en PTF] en la industria es lo que hace que la producción doméstica sea más eficiente, cet. par., y por tanto más competitiva con respecto a productos importados. Por ejemplo, para el sector de vidrio y cerámica, Cárdenas (1987, pp.116) calculó coeficientes de importación ${ }^{23}$ de 43,0 y 13,6 para 1929 y 1934 respectivamente. Esto representa un incremento en la

${ }^{23}$ El coeficiente de importación para el sector $j$, queda definido como el cociente

$$
\text { importaciones }_{j}
$$

VAdomestico $_{j}+$ importaciones $_{j}$ 


\section{CUADRO 3}

CRECIMIENTO PROMEDIO ANUAL DE LA PTF POR SECTOR INDUSTRIAL Y SU CONTRIBUCIÓN AL CRECIMIENTO DE LA PTF AGREGADA 1939-1944

\begin{tabular}{|l|c|c|c|c|c|}
\hline \multicolumn{1}{|c|}{$\begin{array}{c}\text { A } \\
\text { Sector } \\
\text { Industrial }\end{array}$} & $\begin{array}{c}\text { B } \\
\text { Tasa de } \\
\text { Crecimiento } \\
\text { de la PTF }\end{array}$ & $\begin{array}{c}\text { C } \\
\text { Participación } \\
\text { en el Valor } \\
\text { Agregado }\end{array}$ & $\begin{array}{c}\text { D } \\
\text { Contribución } \\
\text { de la PTF al } \\
\text { Crecimiento } \\
\text { (B*C) }\end{array}$ & $\begin{array}{c}\text { E } \\
\text { Participación } \\
\text { acumulada } \\
\text { en el V.A. }\end{array}$ & $\begin{array}{c}\text { E } \\
\text { Contribución } \\
\text { acumulada } \\
\text { de la PTF al } \\
\text { crecimiento }\end{array}$ \\
\hline $\begin{array}{l}\text { Ropa y } \\
\text { Calzado }\end{array}$ & $17,63 \%$ & $4,28 \%$ & $0,75 \%$ & $4,28 \%$ & $0,75 \%$ \\
\hline $\begin{array}{l}\text { Cuero y } \\
\text { Pieles }\end{array}$ & $7,92 \%$ & $1,02 \%$ & $0,08 \%$ & $5,30 \%$ & $0,84 \%$ \\
\hline Textiles & $5,62 \%$ & $26,91 \%$ & $1,51 \%$ & $32,21 \%$ & $2,35 \%$ \\
\hline $\begin{array}{l}\text { Madera y } \\
\text { Muebles }\end{array}$ & $1,32 \%$ & $2,00 \%$ & $0,03 \%$ & $34,21 \%$ & $2,37 \%$ \\
\hline $\begin{array}{l}\text { Alimentos } \\
\text { y Bebidas }\end{array}$ & $-0,83 \%$ & $33,03 \%$ & $-0,27 \%$ & $67,24 \%$ & $2,10 \%$ \\
\hline Construcción & $-0,89 \%$ & $3,13 \%$ & $-0,03 \%$ & $70,37 \%$ & $2,07 \%$ \\
\hline Química & $-1,10 \%$ & $6,65 \%$ & $-0,07 \%$ & $77,02 \%$ & $2,00 \%$ \\
\hline $\begin{array}{l}\text { Cerámica } \\
\text { y Vidrio }\end{array}$ & $-1,73 \%$ & $1,47 \%$ & $-0,03 \%$ & $78,49 \%$ & $1,97 \%$ \\
\hline $\begin{array}{l}\text { Imprenta y } \\
\text { Publicidad }\end{array}$ & $-2,05 \%$ & $2,81 \%$ & $-0,06 \%$ & $81,30 \%$ & $1,92 \%$ \\
\hline Papel & $-3,56 \%$ & $2,07 \%$ & $-0,07 \%$ & $83,37 \%$ & $1,84 \%$ \\
\hline Fundición & $-4,21 \%$ & $11,85 \%$ & $-0,50 \%$ & $95,22 \%$ & $1,34 \%$ \\
\hline Tabaco & $-9,08 \%$ & $4,78 \%$ & $-0,43 \%$ & $100,00 \%$ & $0,91 \%$ \\
\hline
\end{tabular}

Fuente: cálculos propios utilizando datos censales.

sustitución de importaciones ${ }^{24}$ de cerca del 116 por cien -23 por cien en promedio por año ${ }^{25}$. Sin embargo, debe recordarse -de la información presentada en el Cuadro 1- que fue precisamente durante este periodo (1929-1934) cuando el sector de vidrio y cerámica tuvo un avance en productividad muy importante, del 13,14 por cien en promedio anual. Ahora bien, utilizando los estimadores de Cárdenas, en 1939 el sector de vidrio y cerámica presentó un coeficiente de importación de 15,4,

${ }^{24}$ Nótese que un incremento porcentual en el coeficiente de importación representa una reducción del proceso de sustitución de importaciones -el coeficiente de sustitución de importaciones cae-, y viceversa.

${ }^{25}$ Cabe aclarar aquí que nos interesa obtener las tasas promedio anuales, y tradicionalmente éstas se obtienen como media geométrica. Cuando las tasas de cambio quinquenales son negativas, el cálculo del promedio anual a través de la forma tradicional no conduce a resultados sensatos en cuanto a interpretación. Por esta razón, la forma en que se lleva a cabo el cálculo de tasas de crecimiento quinquenal es a través de una diferencia logarítmica, por lo que el promedio anual se obtiene dividiendo la diferencia logarítmica entre cinco. 
lo cual implica una caída en el proceso de sustitución de importaciones de 1934 a 1939 y que va de la mano con la caída en productividad (-2,15 por cien en promedio anual) durante el mismo período, tal y como se muestra en el Cuadro 2. Las estimaciones de este cuadro también muestran que los tres sectores industriales que experimentaron los mejores resultados en cuanto a productividad se refiere fueron los sectores de alimentos y bebidas, fundición y textil, con tasas de crecimiento promedio anual en PTF del 6,86, 6,45 y 4,55 por cien respectivamente. Estos tres sectores juntos tuvieron una participación en el producto total de la industria de cerca del 64 por cien. Adicionalmente, la información contenida en el Cuadro 2 nos muestra que aproximadamente el 90 por cien de la industria manufacturera experimentó avances en productividad, mientras que el restante 10 por cien presentó un retroceso. Resulta también importante señalar que la industria manufacturera pudo haber alcanzado un 4,20 por cien de avance en productividad -crecimiento de la PTF- durante el período 1934-39 si hubiera sido posible eliminar de la actividad económica a aquellos sectores con contribuciones negativas, es decir, si hubiera sido posible eliminar a los tres sectores no productivos -cuero y pieles, vidrio y cerámica y tabaco. Por otra parte, puede notarse en la información del cuadro que, con sólo cuatro de los sectores más productivos, se podría haber logrado un crecimiento en PTF del 3,7 por cien. El Gráfico 2 muestra el correspondiente diagrama de productividad sunrise para el período 1934-1939, resultado de la información presentada en el Cuadro 2. Para el último quinquenio analizado (1939-1944), la tasa de crecimiento promedio anual del PIB resultó ser del 9,2 por cien en términos reales para la muestra de 12 sectores de la industria manufacturera. Sin embargo, para este mismo periodo se observa una tasa de crecimiento promedio anual de la PTF bastante pequeña: 0,91 por cien, lo que significa que solamente el 9,9 por cien de la tasa de crecimiento (promedio anual) del producto es explicada por avances en productividad [o reducción en costos reales] en la industria manufacturera. Es decir, el avance en productividad experimentado durante el periodo es magro (véase Cuadro 3), y principalmente puede atribuirse a la Segunda Guerra Mundial. En el cuadro 3 puede observarse también que solamente cuatro sectores de la industria manufacturera experimentaron crecimiento positivo en PTF, los cuales representan apenas el 34 por cien de la industria, mientras que el resto de la industria presentó retroceso en productividad.

Resulta interesante notar que, durante este quinquenio 1939-1944, la industria pudo haber alcanzado una tasa de crecimiento promedio anual en la PTF del 2,37 por cien, si hubiera sido posible eliminar las contribuciones negativas de los sectores no productivos -que en este caso representan aproximadamente el 66 por cien de la industria manufacturera-, los cuales actuaron como lastre sobre la productividad total de la industria. El Gráfico 3 muestra el correspondiente diagrama de productividad para el período 1939-1944. 


\section{GRÁFICO 1}

DIAGRAMA DE PRODUCTIVIDAD SUNRISE

PARA LA INDUSTRIA MANUFACTURERA, 1929-1934

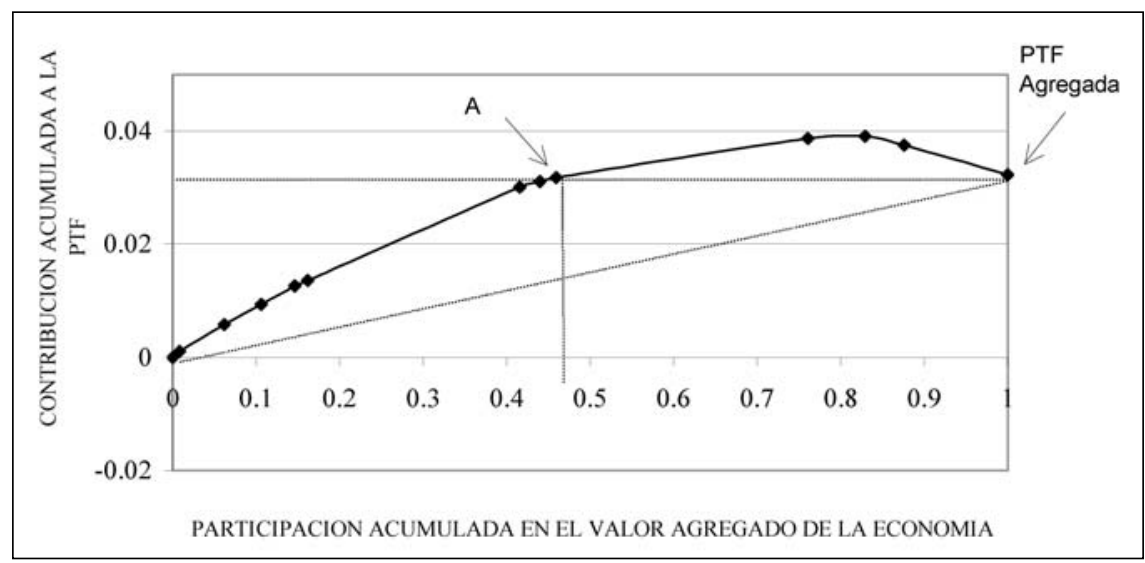

GRÁFICO 2

DIAGRAMA DE PRODUCTIVIDAD SUNRISE

PARA LA INDUSTRIA MANUFACTURERA, 1934-1939

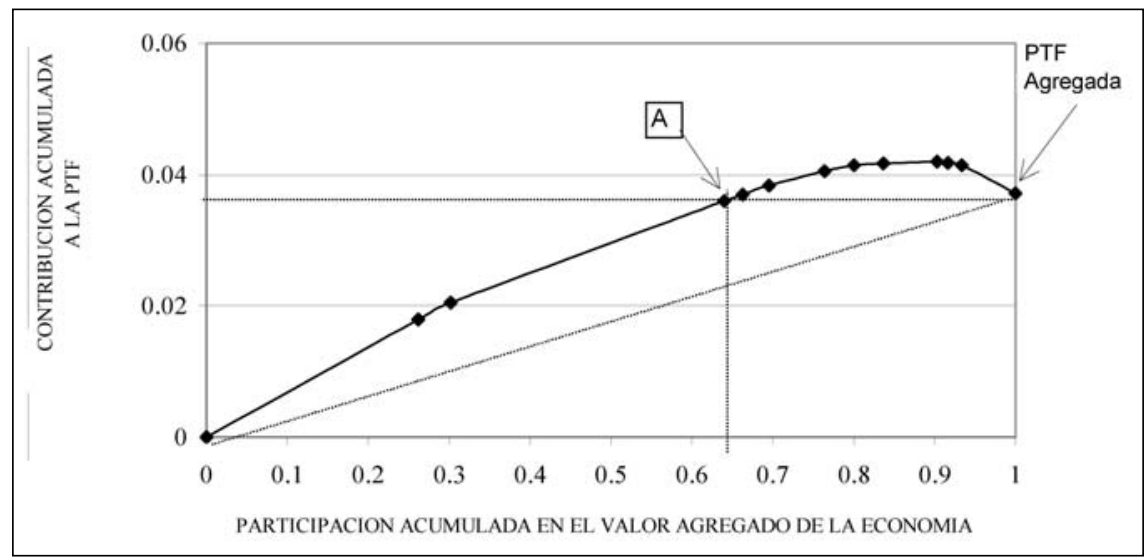




\section{GRÁFICO 3}

DIAGRAMA DE PRODUCTIVIDAD SUNRISE PARA LA INDUSTRIA MANUFACTURERA, 1939-1944

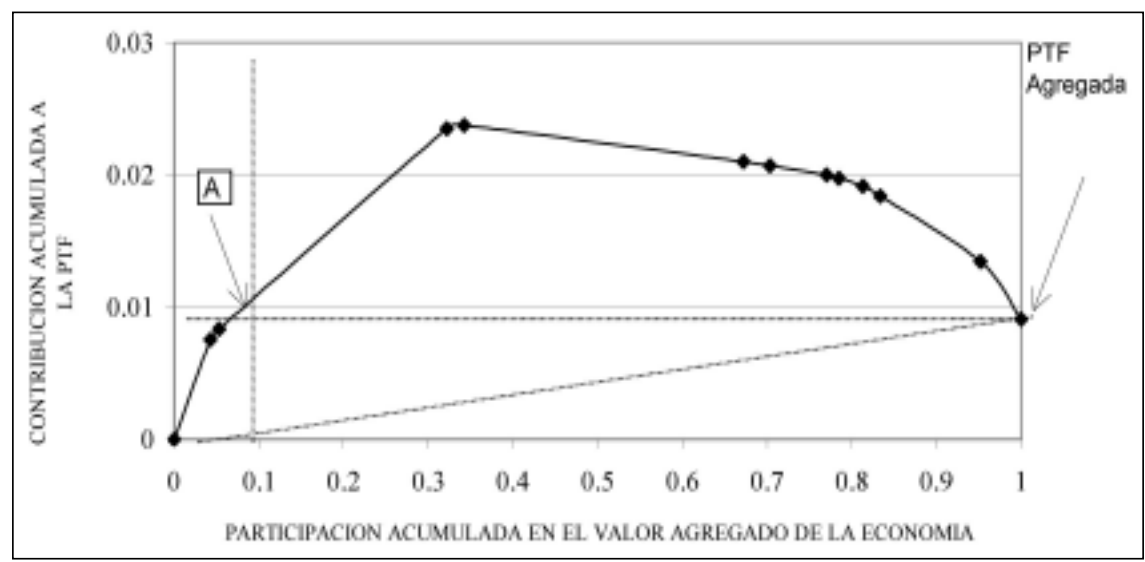

\section{PROCESO DE SUSTITUCIÓN DE IMPORTACIONES Y CRECIMIENTO DE LA PRODUCTIVIDAD}

Las estimaciones obtenidas de las tasas de crecimiento de la productividad nos permitirán ahora abordar el tema correspondiente al segundo objetivo del presente trabajo de investigación, que es el de proporcionar evidencia que dé soporte a la hipótesis de que el proceso de sustitución de importaciones experimentado por la economía mexicana en el período 1929-1939 fue en gran medida causado por un avance sustancial de la PTF. Para proporcionar la evidencia mencionada, haremos uso de las estimaciones de los coeficientes de importación presentadas por Cárdenas ${ }^{26}$. El Cuadro 4 muestra un resumen de las tasas de crecimiento promedio anual de la PTF y de los coeficientes de sustitución de importaciones (CSI), estos últimos calculados como el cambio porcentual en el coeficiente de importación. Un signo negativo en el coeficiente de importación implica que el sector correspondiente experimentó un aumento en el proceso de sustitución de importaciones. Similarmente, un cambio porcentual positivo en el coeficiente de importación significa un retroceso en el proceso de sustitución de importaciones.

\footnotetext{
${ }^{26}$ Cárdenas (1987, pp. 116)
} 
Como puede observarse en el Cuadro 4, la mayoría de los sectores industriales muestran que el avance en la PTF está negativamente correlacionado con el cambio porcentual en el coeficiente de importación.

Con el objeto de proporcionar una prueba de mayor formalidad acerca del efecto que el crecimiento en la PTF tiene sobre el fenómeno de sustitución de importaciones, utilizamos un modelo de regresión simple, donde tomamos el coeficiente de sustitución de importaciones para cada industria como la variable dependiente en función de la tasa de crecimiento de la $\mathrm{PTF}^{27}$, ambos expresados en tasas pro-

${ }^{27}$ El lector podría cuestionarse a la validez, desde el punto de vista econométrico, de la utilización de una sola ecuación para la estimación y prueba de la relación existente entre la PTF y el coeficiente de sustitución de importaciones. Específicamente, pudiera pensarse en un problema de simultaneidad entre $c s i$ y ptf, el cual, de existir, implicaría que la variable $p t f$ es endógena en la ecuación planteada para csi, y por tanto los estimadores serían sesgados e inconsistentes. Sin embargo, podemos mencionar que no se tiene conocimiento de un modelo (económico) teórico que fundamente que la ptf y el csi se determinen de forma simultánea. Más aún, teóricamente resulta difícil justificar una ecuación en la que el csi explique o determine la $p t f$. Pudiera pensarse también en establecer una ecuación para $p t f$ que complemente un sistema de dos ecuaciones simultáneas y en la que no necesariamente quede incluida la variable csi como explicativa. Sin embargo, hasta la fecha, en la literatura relacionada con estimaciones econométricas de la PTF, la forma en que esta variable es estimada es siempre como el término de error (estimado) proveniente de una ecuación de crecimiento del producto que se estipula en función del crecimiento en los factores de producción (véase, por ejemplo, López-Córdoba, 2003). Por tanto, no existe estrictamente un modelo econométrico en el que se establezca como variable dependiente a la PTF en función de un grupo de variables explicativas. Por otra parte, debemos mencionar que, si bien es cierto que el concepto de variables explicativas exógenas y endógenas se ubica básicamente en el contexto de sistemas de ecuaciones, el término «variable explicativa endógena» ha evolucionado para cubrir cualquier caso en el que una variable explicativa esté correlacionada con el error en la ecuación de regresión. Es decir, aun teniendo un modelo uniecuacional para $c s i$, cabe el cuestionamiento respecto a la posibilidad de que ptf sea endógena en la ecuación [4]. A este respecto debe decirse que existen varios motivos por los cuales la variable explicativa -en este caso ptf- pudiera estar correlacionada con el término de error. El primer motivo se refiere al error de medición en la variable explicativa, lo cual crea sesgo e inconsistencia en los estimadores de mínimos cuadrados ordinarios. Este problema generalmente se presenta cuando se utiliza una variable «proxy» para la variable explicativa ante la no disponibilidad de información sobre esta última. Por supuesto, consideramos que éste no es el caso de la ptf. El segundo motivo por el cual el término de error puede estar correlacionado con $\mathrm{ptf}$ es la omisión de alguna variable relevante que se encuentre a su vez correlacionada con ptf. Respecto a este punto, cabe señalar que otra de las variables que teóricamente es determinante del csi es la tasa de devaluación real, variable que se encuentra omitida en la ecuación de regresión [4], pues solamente contamos con dos observaciones en el tiempo en nuestro panel de datos, e incluirla sería como incluir un efecto fijo -para dos períodos. A pesar de la omisión de la tasa de devaluación real como variable explicativa en el modelo para csi, no debemos olvidar que el sesgo por omisión de variables es cero si la variable omitida -aun siendo relevante- no está correlacionada con la(s) variable(s) explicativa(s) incluida(s). Para nuestro caso, resulta poco obvio que ptf esté correlacionado con la tasa de devaluación real, por lo que se hace necesaria la prueba de significación estadística del coeficiente en la regresión de la tasa de devaluación real en función de ptf. El resultado de la prueba nos indica que el $p$ valor del coeficiente asociado a la pendiente de esta regresión es 0,18 , lo que implica 
medio anuales. El fundamento teórico que justifica la relación entre el crecimiento de la PTF y el proceso de sustitución de importaciones puede encontrarse en el argumento de que, ceteris paribus, un aumento en la PTF trae como resultado una mejora en la posición comercial del país en cuestión -por lo menos en los sectores en los que se observa el aumento en la PTF. Es decir, un avance en productividad puede verse reflejado en precios más competitivos en comparación con los del exterior, estimulando así el proceso de sustitución de importaciones. Por otra parte, cabe aquí mencionar que, si bien es cierto que tradicionalmente se ha considerado que un proceso de sustitución de importaciones es resultado de la política comercial y cambiaria de un país -es decir, de las políticas implementadas para proporcionar protección al mercado interno-, tampoco podemos descartar $a$ priori -al menos teóricamente- la hipótesis de que una mejora en la productividad permita al productor de un bien ofrecer sus productos a mejores precios, captando así una mayor proporción del mercado interno, lo que implica una sustitución de importaciones. Es en este sentido que un proceso de sustitución de importaciones puede concebirse como una variable relacionada con un argumento de oferta como lo es la PTF, aunque indiscutiblemente también está relacionado con argumentos de demanda, como lo son las cuotas y tarifas a la importación, así como el tipo de cambio real.

La regresión se estimó utilizando un panel con doce sectores y dos períodos quinquenales (1929-1934 y 1934-1939), por lo que en total se tienen 24 observaciones de las variables (vid. Cuadro 4). Los resultados para la ecuación estimada son los siguientes:

$$
\begin{gathered}
c s i_{j t}=-0,44998-2,01558 p t f_{j t}[4] \\
(2,0325)(0,3653)
\end{gathered}
$$

donde $c s i_{j t}$ es el cambio porcentual promedio anual en el coeficiente de importación para el sector $j$ en el quinquenio $t$. La variable explicativa $p t f_{j t}$ es la tasa de crecimiento promedio anual en la PTF para el sector $j$ en el quinquenio $t, y$ las

que no es estadísticamente diferente de cero, y por lo tanto no puede rechazarse la hipótesis de que ptf no está correlacionada con la variable omitida. Es decir, no hay sesgo por omisión de variable relevante en las estimaciones realizadas en [4]. Otro de los motivos por el que el término de error en la regresión pudiera estar correlacionado con ptf es el denominado problema de sesgo por selección de la muestra (selectivity bias problem), el cual surge principalmente cuando la variable dependiente en el modelo es una variable truncada o censurada (Greene, 2003). Sobra decir que, para el caso que nos ocupa, no resulta sensato pensar en un problema de sesgo por selección. Otra de las razones por las que el lector pudiera cuestionar la validez del modelo estimado en [4], es la causalidad entre la ptf y el csi. Al respecto de este tema, podemos decir que tratar de determinar estadísticamente la causalidad entre las variables csi $y$ $p t f$-realizar la prueba de Granger por ejemplo-, no es posible, dada la naturaleza de los datos con los que se cuenta. En este caso sólo hay dos períodos de tiempo para cada sector. 
observaciones corresponden a los cálculos presentados en los Cuadros 1 y 2, que a su vez se resumen en el Cuadro 4 . Los errores estándar ${ }^{28}$ de los coeficientes estimados se expresan entre paréntesis -véase nota 28 para las pruebas de heteroscedasticidad correspondientes. En particular, para el coeficiente asociado a la variable $p t f$, el valor muestral del estadístico $t$ es de $-5,516$, implicando esto que el coeficiente es estadísticamente diferente de cero a niveles de significación del 5 por cien y del 1 por cien inclusive ${ }^{29}$. Adicionalmente, el coeficiente de determinación $\mathrm{R}^{2}$ fue del 0,58 lo que significa que las variaciones en las tasas de crecimiento de la PTF explican el 58 por cien de las variaciones en el coeficiente de sustitución de importaciones (en torno a su media).

Los resultados de la estimación también confirman el signo esperado para el coeficiente de la pendiente, implicando esto que un incremento en la tasa de crecimiento de la PTF reduce el coeficiente de importación. En otras palabras, un incremento en la productividad incentiva el proceso de sustitución de importaciones. De acuerdo con Cárdenas, el proceso de sustitución de importaciones fue estimulado especialmente por devaluaciones del tipo de cambio $^{30}$ que tuvieron lugar durante los períodos de 1929 a 1933 y de 1938 a 1940. Sin embargo, los resultados de nuestro análisis (véase el Cuadro 4) mostraron que seis sectores de la industria manufacturera experimentaron un retroceso en el proceso de sustitución de importaciones durante el período 1934-1939, y uno en el período 1929-1934. Si la política cambiaria hubiera sido la causa principal del proceso de sustitución de importaciones para la época, se habría observado entonces un incremento en la sustitución de importaciones para todos los sectores analizados. Pero esto no es lo que se observa, por lo que puede argumentarse que hay otros factores determinantes del proceso de sustitución de importaciones, y con el análisis realizado se presenta evidencia de que el crecimiento de la PTF es uno de estos determinantes.

${ }^{28}$ Para propósitos de estimación, los datos fueron agrupados en forma de panel, por lo que se realizaron pruebas de heteroscedasticidad para verificar que los errores estándar utilizados son los adecuados. En este caso, se realizaron las pruebas de White y de Goldfeld-Quandt. Los resultados de ambas pruebas mostraron que no hay suficiente evidencia para rechazar la hipótesis nula de homoscedasticidad en los errores. Para la prueba de White, el valor muestral del estadístico de prueba (F) resulta ser de 0,8322 con un $p$ valor de 0,4489; similarmente, para la prueba Goldfeld-Quandt, el valor muestral del estadístico de prueba es de 1,06456 con un $p$ valor correspondiente de 0,4642 , con lo que se concluye que en ambas pruebas no hay evidencia para rechazar la hipótesis nula de no heteroscedasticidad.

${ }^{29}$ Nótese que la ordenada en el origen de la regresión no es estadísticamente significativa.

${ }^{30}$ Tomando datos de Cárdenas (1987, cuadro A4.10, p. 239), la devaluación nominal registrada durante el quinquenio 1930-1934 fue del 62,79 por cien -implicando un 10,24 por cien en promedio anual. Las mayores tasas de devaluación para este quinquenio se dieron en 1931 y 1932: 17,33 y 19,7 por cien, respectivamente. Para el período 1935-1939, la devaluación nominal fue del 48,29 por cien -lo que significa un 8,2 por cien en promedio anual. Para este quinquenio las devaluaciones se registraron básicamente en 1938 y 1939: 25,56 y 14,89 por cien respectivamente. 
Como complemento al análisis anterior, se tratará de identificar la influencia que el proceso de sustitución de importaciones tuvo sobre el crecimiento en valor agregado de la industria manufacturera durante los quinquenios 1929-1934 y 1934 1939. Para esto haremos uso nuevamente de los coeficientes de importación proporcionados por Cárdenas.

Lo primero que hay que mencionar es que el coeficiente de correlación muestral entre los cambios porcentuales en el coeficiente de importación ${ }^{31}\left(c s i_{j t}\right)$ y los cambios porcentuales en el valor agregado del sector correspondiente $\left(v a_{j t}\right)$ para el mismo quinquenio, resulta ser de $-0,318$, lo que nos indica que, aunque el signo de la relación es correcto -ya que es de esperar que mientras menor sea el cambio porcentual en el coeficiente de importación, mayor sea el crecimiento en VA-, el grado de asociación lineal entre estas dos variables es bastante bajo.

Además de calcular el coeficiente de correlación, podría pensarse en estimar una regresión simple para determinar la influencia que las variaciones en el coeficiente de importación tienen sobre el crecimiento en el VA. Sin embargo, sabemos que el crecimiento en el VA está determinado por muchos otros factores -entre ellos el crecimiento de la PTF- y no solamente por el proceso de sustitución de importaciones experimentado por el sector. El incluir como variable explicativa únicamente a la variable $c s i_{j t}$ crearía entonces lo que conocemos como sesgo por omisión de variable relevante, especialmente si se toma en cuenta que una de las variables omitidas (crecimiento de la PTF) está correlacionada con $c s i_{j t}$.

Tomando, entonces, en consideración que el crecimiento en la productividad es un determinante importante del crecimiento del VA-por definición-, y con el objetivo de poder estimar la influencia que el proceso de sustitución de importaciones tiene sobre el cambio porcentual en VA, se lleva a cabo la estimación del modelo siguiente ${ }^{32}$ :

${ }^{31}$ Cabe mencionar que, para evitar sesgo en los resultados, se excluyó de la muestra al sector de fundición, ya que las variaciones porcentuales en el VA real por quinquenio resultaron bastante grandes: -62,35 y 165,64 por cien respectivamente.

${ }^{32}$ El lector podría sugerir que, para propósitos de inferencia, la especificación del modelo es incorrecta, ya que las variables explicativas ptf y csi se encuentran correlacionadas linealmente, por lo que el problema de colinealidad está presente. Sin embargo, recordemos que, en la regresión estimada con anterioridad para $c s i$ en función de $p t f$, el $\mathrm{R}^{2}$ resultó ser de 0,58 , con lo que puede pensarse que utilizar ambas variables como explicativas no crea problemas con los errores estándar de los coeficientes, y por lo tanto las pruebas de hipótesis basadas en estos errores estándar tienen validez estadística. Por otra parte, al igual que en el caso de la ecuación de regresión [4], podría cuestionarse la correcta especificación del modelo en [5]. Es decir, puede dudarse sobre la validez de los resultados de la estimación al sospecharse la presencia de un problema de endogeneidad, el cual puede surgir por cualquiera de las causas explicadas en la nota 27. A este respecto diremos que se realizó la prueba de endogeneidad de Hausman (véase Wooldridge, 2002) para csi en la ecuación [5]. Para modelos uniecuacionales la prueba de Hausman puede realizarse en el contexto de estimación con variables instrumentales. En este caso, la variable instrumental utilizada para csi es la tasa de devaluación real, ya que está (parcialmente) correlacionada con csi. La hipótesis nula en este tipo de prueba es que la variable $c s i$ es exógena. El $p$ valor asociado al valor muestral del estadístico de prueba utilizado en la prueba de endogeneidad de Hausman (Wooldridge, 2002, pp 119-121), resultó ser 0,16, por lo que no hay evidencia para rechazar la hipótesis nula de exogeneidad para la variable csi. 


\section{CUADRO 4}

\section{SUSTITUCIÓN DE IMPORTACIONES Y CRECIMIENTO DE LA PTF}

\begin{tabular}{|l|c|c|c|c|}
\hline & \multicolumn{2}{|c|}{$\mathbf{1 9 3 4 - 1 9 3 9}$} & \multicolumn{2}{c|}{$\mathbf{1 9 2 9 - 1 9 3 4}$} \\
\cline { 2 - 5 } Sector Industrial & $\begin{array}{c}\text { Coeficiente } \\
\text { de } \\
\text { Importación } \\
\text { (variación } \\
\text { porcentual } \\
\text { promedio } \\
\text { anual) }\end{array}$ & $\begin{array}{c}\text { Tasa de } \\
\text { crecimiento } \\
\text { promedio } \\
\text { anual de la } \\
\text { PTF }\end{array}$ & $\begin{array}{c}\text { Coeficiente } \\
\text { de } \\
\text { Importación } \\
\text { (variación } \\
\text { porcentual } \\
\text { promedio } \\
\text { anual) }\end{array}$ & $\begin{array}{c}\text { Tasa de } \\
\text { crecimiento } \\
\text { promedio } \\
\text { anual de la } \\
\text { PTF }\end{array}$ \\
\hline Textiles & $-14,63 \%$ & $6,50 \%$ & $-3,65 \%$ & $4,55 \%$ \\
\hline Ropa & $-8,66 \%$ & $0,49 \%$ & $-6,01 \%$ & $3,23 \%$ \\
\hline Alimentos y Bebidas & $-5,17 \%$ & $2,30 \%$ & $-23,86 \%$ & $6,86 \%$ \\
\hline Tabaco & $-38,92 \%$ & $8,98 \%$ & $13,86 \%$ & $-6,40 \%$ \\
\hline Química & $-10,41 \%$ & $8,11 \%$ & $\mathbf{0 , 5 2 \%}$ & $\mathbf{0 , 4 9 \%}$ \\
\hline Cuero y Pieles & $-21,57 \%$ & $6,68 \%$ & $11,49 \%$ & $-1,30 \%$ \\
\hline Imprenta y Publicidad & $-\mathbf{0 , 3 1 \%}$ & $-3,40 \%$ & $\mathbf{5 , 7 5 \%}$ & $\mathbf{4 , 1 6 \%}$ \\
\hline Vidrio y Cerámica & $-23,02 \%$ & $13,14 \%$ & $2,49 \%$ & $-2,15 \%$ \\
\hline Construcción & $-14,19 \%$ & $4,25 \%$ & $-4,79 \%$ & $0,68 \%$ \\
\hline Madera y Muebles & $-17,87 \%$ & $7,85 \%$ & $-3,63 \%$ & $2,51 \%$ \\
\hline Papel & $-6,83 \%$ & $3,45 \%$ & $\mathbf{6 , 5 1 \%}$ & $\mathbf{4 , 4 1 \%}$ \\
\hline Fundición & $1,93 \%$ & $-4,17 \%$ & $-6,38 \%$ & $6,45 \%$ \\
\hline
\end{tabular}

[5]

Es pertinente aclarar en este momento que, si bien es cierto que el VA está determinado tanto por elementos de oferta como de demanda, el modelo planteado en la ecuación [5] tiene sus limitaciones. Específicamente debe mencionarse que la PTF -como variable de oferta- y el proceso de sustitución de importaciones -como variable de demanda- no representan a todas las variables que influyen sobre el VA. Sin embargo, nuestro interés se centra en tratar de identificar la influencia que el proceso de sustitución de importaciones hubiera tenido en el crecimiento del VA sectorial de la época de estudio, y con ese objetivo presentamos el análisis econométrico propuesto. Por otra parte, el lector puede argumentar que el modelo planteado en [5] presenta problemas de endogeneidad. Para descartar la existencia de este problema se realizó la prueba de Hausman cuyos resultados se especifican en la nota 32 .

Los resultados de la estimación seresentan a continuación en el Cuadro 5: 


\section{CUADRO 5}

RESULTADOS DE LA ESTIMACIÓN DEL MODELO [5]

\begin{tabular}{|l|c|c|c|c|}
\hline \multicolumn{1}{|c|}{ Variable } & Coeficiente & Error Std. & Estadístico t & Prob. \\
\hline ptf & 0,935122 & 0,337465 & 2,771015 & 0,0122 \\
\hline csi & 0,125368 & 0,120158 & 1,043359 & 0,3099 \\
\hline C & 3,276983 & 1,191656 & 2,749940 & 0,0127 \\
\hline
\end{tabular}

Como puede observarse, el crecimiento en la productividad (ptf) definitivamente explica el crecimiento en el VA. Sin embargo, el coeficiente asociado a csi no solamente presenta el signo equivocado, sino que, además, éste no resulta ser estadísticamente diferente de cero, por lo que puede concluirse que, para el período y sectores analizados, el proceso de sustitución de importaciones -crecimiento en el coeficiente de importación- no resultó determinante, estadísticamente hablando, en el crecimiento del VA. El coeficiente de determinación para el modelo estimado es de 0,36.

Considerando las limitaciones del modelo planteado en [5], cabe señalar que las conclusiones obtenidas con datos a nivel de sector sobre la relevancia que el crecimiento en la PTF tiene en la explicación del crecimiento en el VA no descansan únicamente en los resultados de esta regresión. Las conclusiones son congruentes con los resultados obtenidos en el análisis realizado al final del apartado 3. Recordemos que en ese mismo apartado se mencionó que, para el período 19291934, el crecimiento promedio anual del VA del total de la industria manufacturera fue del 4,2 por cien, mientras que la correspondiente tasa de crecimiento promedio anual en la PTF fue del 3,23 por cien, implicando esto que 76,9 por cien del crecimiento en VA es explicado por el crecimiento en la PTF. De igual manera, para el período 1934-1939, el VA de la industria manufacturera creció 7,9 por cien en promedio anual, mientras que el correspondiente crecimiento en PTF fue de 3,72 por cien, lo que indica que el 47,08 por cien del crecimiento en el VA es explicado por crecimiento en la PTF.

\section{CONCLUSIONES}

Utilizando datos desagregados a nivel de sector y aplicando el método de los dos deflactores de Harberger, el presente trabajo de investigación ha examinado en detalle la evolución de la productividad total de los factores en doce sectores que forman la mayor parte de la industria manufacturera en México para el período 1929-1944. A lo largo del análisis se ha mostrado claramente la relevancia que el avance en la productividad tiene como fuente de crecimiento de la economía, y 
en específico, el análisis presenta evidencia de que el avance en la productividad total de los factores fue la causa principal del crecimiento observado en la industria manufacturera durante el período de estudio, y no así la sustitución de importaciones, ya que un incremento en productividad genera un cambio porcentual negativo en el coeficiente de importación -es decir, un incremento en el proceso de sustitución de importaciones-, y viceversa, pero las variaciones en el coeficiente de importación, de acuerdo con el modelo estimado, no explican el crecimiento en valor agregado observado en la época. A pesar de la política cambiaria implementada de 1929 a 1933 y de 1938 a 1940 -que, de acuerdo con Cárdenas, fue el principal motor del proceso de sustitución de importaciones observado-, los resultados de nuestro análisis mostraron que seis sectores de la industria manufacturera experimentaron un retroceso en el proceso de sustitución de importaciones durante el período 1934-1939, y uno en el período 1929-1934. Si la política cambiaria hubiera sido la causa principal del proceso de sustitución de importaciones para la época, se habría observado entonces un incremento en la sustitución de importaciones para todos los sectores analizados. Sin embargo, esto no es lo que se observa, por lo que puede argumentarse que hay otros factores determinantes del proceso de sustitución de importaciones, y en el presente artículo se ha presentado evidencia de que el avance en productividad es uno de estos determinantes. Si bien se ha demostrado que el crecimiento en la PTF observado durante los años treinta en la industria manufacturera fue un componente importante de la tasa de crecimiento de la industria, hay que mencionar que el crecimiento de la PTF va siempre acompañado de un incremento en la tasa de rendimiento del capital, lo que a su vez se traduce en un incremento en la inversión. Siguiendo esta línea, los resultados del presente estudio concuerdan con otro análisis de Enrique Cárdenas ${ }^{33}$ en el que se menciona que el crecimiento económico de 1940 a 1960 -el llamado «milagro mexicano»-, obedece a la gran acumulación de capital que hubo en los años treinta ${ }^{34}$. Esta gran acumulación de capital no habría sido posible si la tasa de rendimiento del capital no se hubiera incrementado en este período, y no hay que olvidar que esto último es consecuencia del crecimiento en la PTF, entre otras cosas.

\section{FUENTES}

Primero, Segundo, Tercer y Cuarto Censos Industriales (1930, 1935,1940 y 1945). México: Secretaría de Economía.

${ }^{33}$ Cárdenas (1982).

${ }^{34}$ Cárdenas (1987, pp. 137-142) y (1994, pp. 59-60) menciona que en buena medida el crecimiento industrial se debió a la fuerte inversión tanto privada como pública, siendo la primera especialmente orientada a infraestructura (construcción de red de carreteras y ferrocarriles). 


\section{BIBLIOGRAFÍA}

Abramovitz, M. (1962): «Economic Growth in the United States». American Economic Review 52 (4), pp. 762-782.

Angulo, J. y Guillermo, S. (2004): «Un análisis del desempeño de la industria manufacturera en México considerando el problema de datos perdidos: 1929-1934». México: Centro de Investigaciones y Estudios de Posgrado, Facultad de Economía BUAP. Documento de Trabajo.

Banco de México (1994): «Encuesta de Acervos y Formación de Capital: 1960-1994». México: Banco de México. Dirección General de Investigación Económica. Gerencia de Información Económica. Serie Documentos Internos. Reportes Metodológicos.

CÁrdenas E. (1978): «El Crecimiento Económico en México: 1950-1975». México: ITAM. Tesis de Licenciatura.

- (1982): «The Great Depression and Industrialization: The Case of Mexico». México: El Colegio de México, Centro de Estudios Económicos. Documentos de Trabajo No. 10.

- (1987): La Industrialización Mexicana Durante la Gran Depresión. México: El Colegio de México.

- (1994): La Hacienda Pública y la Política Económica, 1929-1958. México: El Colegio de México / FCE.

Сно, М. (2000): «The Asian Miracle and the Asian Economic Crisis: A disaggregated analysis of productivity growth». Los Ángeles, Ca: UCLA. Ph D. Dissertation.

Denison, E. (1967): Why growth rates differ: Post-war experience in nine western countries. Washington, D.C.: Brookings Institution.

Goodman, L. (1968): «The Analysis of Cross-Classified Data: Independence, QuasiIndependence, and Interactions in Contingency Tables With or Without Missing Entries». Journal of The American Statistical Association 63 (324), pp. 1091-1131.

Greene, W. (2003): Econometric Analysis. Nueva Jersey: Prentice Hall, $5^{a}$ edición.

Griliches, Z. (1963): «The Sources of Measured Productivity Growth: United States Agriculture, 1940-60». Journal of Political Economy 71(4), pp. 331-346.

HABER, S. (1989): Industry and Underdevelopment. The Industrialization of Mexico, 18901940. Stanford: Stanford University Press.

Harberger, A. (1998a): «Studying the Growth Process: A Primer». Papel presentado a la Conferencia Capital Formation and Economic Growth. Stanford, CA: Hoover Institution, diciembre 1998.

— (1998b): «A Vision of the Growth Process». The American Economic Review 88 (1), pp. 1-31.

Hernández Laos, E. (1985): La productividad y el desarrollo industrial en México. México: Fondo de Cultura Económica.

Jorgenson, D. W. (1967): «The Explanation of Productivity Change». Reimpresión de Review of Economic Studies XXXIV(3), No. 99

López-CóRdoBA, E. (2003): «Nafta and Manufacturing Productivity in México». Economía 3 (2), pp. 55-98.

RoBLEs, E. (1997): «An Exploration into the Sources and Causes of Economic Growth in the United States and Fourteen Latin American Countries». Los Ángeles, Ca: UCLA. Ph.D. Dissertation. 
Solís, L. (1987): La Realidad Económica Mexicana: retrovisión y perspectivas. México: Ed. Siglo XXI.

Torre, L. (2000): «Productivity and Profitability in the Mexican Manufacturing Sector: 1984-1994». Los Ángeles, Ca: UCLA. Ph.D. Dissertation.

Villalpando Hernández, L. H. y Fernández Morán, J. (1987): «Encuesta de acervos y formación de capital: 1960-1987». México: Banco de México.

Wooldridge, J. (2002): Econometric Analysis of Cross Section and Panel Data. Londres: The MIT Press.

- (2003): Introductory Econometrics: A Modern Approach. Ohio: Thomson Learning, Sowth Western, $2^{\text {a }}$ edición.

\section{APÉNDICE}

\section{Estimación del gasto Total en Materias Primas}

El gasto total en materias primas $\left(r m_{j t}\right)$ se calculó sumando a la cantidad que aparece como «materias primas» en los censos los gastos en electricidad, lubricantes, combustible y otros gastos -que incluye rentas, costos de transacción, seguros, etc. La razón por la que se suman los gastos mencionados al concepto de materias primas reportado por los censos es que éstos representan una parte importante de todos los materiales que se necesitan en el proceso de producción en la mayoría de las industrias. No podemos, entonces, ignorarlos cuando se lleva a cabo la estimación de los costos de producción. Sin embargo, debe mencionarse que la información relacionada con los gastos en los rubros de electricidad, lubricantes, combustibles y otros, no se encuentra disponible para todos los sectores en los censos de nuestro interés. Específicamente, el censo de 1944 es el que proporciona la información más completa a este respecto. Por tanto, la proporción que los gastos en electricidad, lubricantes, combustibles y otros representa del gasto en materias primas reportado en el censo de 1944 será tomada -como constante por sector- para estimar los gastos correspondientes en los rubros mencionados de los censos de 1929, 1934 y 1939.

\section{Corrección de datos para el Censo de 1929}

Los dos sectores con mayor participación en el VA agregado de la industria manufacturera -más del 50 por cien- durante los tres quinquenios analizados fueron el sector de alimentos y bebidas y el sector textil, cuyas participaciones en el PIB agregado de la industria manufacturera -que incluye 12 industrias en la muestra-, resultan ser del 36 y 29,13 por cien respectivamente en el período 1929-1934, del 26,21 y 33,85 por cien en el período 1934-1939, y 33,06 y 26,91 por cien para el 
período 1934-1939. Al realizar las estimaciones de PTF con los datos directamente tomados de los censos, se observaron resultados para el período 1929-1934 que no son consistentes con la teoría según la cual el proceso de industrialización en México tuvo sus raíces en el período post Gran Depresión. En particular, el sector de alimentos y bebidas, presentó un retroceso importante en productividad (-5,95 por cien) en el primer quinquenio, con lo que este sector se ubicaría en el último lugar en desempeño para el período 1929-1934. Sin embargo, llama la atención que, para el quinquenio 1934-1939, el sector de alimentos y bebidas ocupa el primer lugar en la lista de avances en productividad, con un 6,86 por cien de crecimiento promedio anual en la PTF (véase Cuadro 2). ¿Qué es lo que ocasionó tal cambio en la productividad del sector alimentos y bebidas? ¿Por qué las estimaciones presentan en general un mal desempeño en PTF de los sectores para el primer y tercer quinquenio, cuando este período se supone que fue el detonante del proceso de industrialización en México? El afán de responder a estas preguntas nos lleva a profundizar aún más en el análisis de los datos, concentrándonos así en datos más desagregados para cada uno de los sectores. El análisis a nivel de subsector nos revela que tres subsectores del sector alimentos y bebidas no reportaron el rubro de materias primas utilizadas en el proceso productivo en el censo realizado en 1929. Los mencionados subsectores son: molinos de nixtamal, beneficiadoras de café y bebidas hechas a base de agave. Si bien la información sobre materias primas es omitida en 1929, para los censos subsecuentes ésta si se encuentra reportada, pero la consecuencia de ignorar esta falta de información resulta en la sobreestimación del valor agregado de la industria alimenticia y (peor aún) su correspondiente tasa de rendimiento de capital para 1929. La sobreestimación del VA en el sector se torna en un problema aún más importante si consideramos que estos tres subsectores produjeron el 22,63 por cien del total de la producción del sector de alimentos y bebidas en 1929. Esta es precisamente la razón por la que el sector alimentos y bebidas muestra un fuerte retroceso en productividad de 1929 a 1934 cuando se toman los datos agregados y directamente de los censos. Dado que no se cuenta con información sobre materias primas en los subsectores mencionados para 1929, procedemos a realizar una estimación de este rubro utilizando un simple promedio aritmético ${ }^{35}$ de las proporciones que las materias primas representaron dentro del producto total de la industria alimenticia en los tres censos subsecuentes ${ }^{36}$. Una vez realizada la corrección del valor

${ }^{35}$ La utilización del promedio aritmético puede considerarse correcta en este caso, ya que el cociente entre materias primas y producto no presentó mucha variación durante el período de análisis.

${ }^{36}$ Para el subsector de beneficiadoras de café, la proporción promedio de materias primas fue del 72,48 por cien del producto total del subsector. Para el subsector de bebidas a base de agave, la proporción promedio fue del 25,6 por cien, y la correspondiente para molinos de nixtamal es del 73,93 por cien del total de su producción. 
agregado para la industria de alimentos y bebidas, la correspondiente tasa de crecimiento promedio anual de la PTF es de 2,3 por cien para el quinquenio 19291934. Es decir, la corrección del VA de la industria nos indica que, en realidad, ésta observó un avance en productividad, y no un retroceso como indica el análisis cuando se realiza con datos agregados. Una situación similar a la descrita presenta la industria textil, la cual mostró un ligero retroceso en productividad durante 1929-1934 (-0,80 por cien), dando un salto bastante importante para el quinquenio 1934-1939, del 4,55 por cien en avance en productividad promedio anual, si se consideran los datos agregados. Nuevamente, al tratar de encontrar una respuesta al por qué del cambio radical en el desempeño del sector textil, el análisis con un mayor nivel de desagregación en los datos nos lleva a descubrir que dos subsectores, denominados despepitadoras de algodón y desfibradoras de henequén, tampoco reportaron el rubro de materias primas en el censo de 1929, pero sí lo hicieron en los censos subsiguientes. La omisión de la información en este censo se agrava aún más si consideramos que estos dos subsectores representaron el 32,9 por cien de la producción total del sector textil en ese año. Es ésta, pues, la razón por la que el VA y la tasa de rendimiento del capital se encuentran sobreestimadas para 1929 cuando se utilizan los datos agregados tal y como aparecen en la fuente. Al igual que en el caso del sector de alimentos y bebidas, se realizó una estimación de la proporción que las materias primas representan dentro de la producción total, utilizando la información de los tres censos siguientes en la muestra $^{37}$. Por tanto, una vez realizada la corrección del VA de la industria textil, la estimación del avance en productividad para el quinquenio 1929-1934 resulta ser un 6,5 por cien en promedio anual, lo que representa un cambio bastante importante con relación a la estimación arrojada con los datos en bruto.

El tercer sector que presenta el problema de omisión de datos de materias primas en el censo de 1929 es el de madera y muebles. Si bien esta industria tiene una participación relativamente pequeña en la producción total del sector manufacturero -no mayor al 3,6 por cien en los tres quinquenios analizados-, consideramos que es importante la realización de la correspondiente corrección de datos de VA para 1929, con el objeto de lograr estimadores más fiables en lo que a cambios en la PTF se refiere. En este sector, es el subsector de aserraderos -el cual representó un 14,5 por cien de la producción total del sector madera y muebles en 1929- el que no reporta las materias primas utilizadas. La estimación correspondiente de la proporción de materias primas utilizadas es el 37,45 por cien del valor de la producción, y, considerando esta corrección para el cálculo de VA de la industria de la madera, el crecimiento de la PTF es de 7,85 por cien en promedio anual para el período 1929-1934.

${ }^{37}$ Para el subsector del despepite de algodón, la proporción estimada de materias primas fue del 74,53 por cien de la producción total del subsector. Para el subsector de desfibración de henequén, la correspondiente proporción estimada fue de 36,47 por cien. 
Cabe mencionar que, además de las modificaciones en la PTF para los sectores mencionados, las correcciones realizadas al VA de los tres subsectores indudablemente afectan al VA de toda la industria manufacturera ${ }^{38}$, por lo que las correspondientes participaciones por sector en el VA total de la industria se ven también afectadas. El Cuadro 1 muestra, así, las estimaciones corregidas para el crecimiento de la PTF en el quinquenio 1929-1934 por sector, y sus correspondientes participaciones en el VA total, siendo ambos información relevante en la elaboración del diagrama de productividad Sunset-Sunrise.

${ }^{38}$ Los nueve sectores restantes incluidos en el análisis sí reportaron el rubro de materias primas en el censo de 1929. 